\title{
Design of a Matched Index of Refraction Facility for Flow Studies with Mixing and Complex Geometries
}

\author{
Abdullah G. Weiss ${ }^{1 *}$, Paul J. Kristo ${ }^{2}$, Mark L. Kimber ${ }^{1,2}$ \\ ${ }^{1}$ Department of Nuclear Engineering, Texas A\&M University, College Station, TX 77840, USA \\ ${ }^{2}$ Department of Mechanical Engineering, Texas A\&M University, College Station, TX 77840, USA
}

\begin{abstract}
A matched index of refraction facility has been constructed at Texas A\&M University to enable optically transparent measurements in a test section capable of laminar, transition, and turbulent flow regimes. The index matching is achieved by recirculating Drakeol ${ }^{\circledR} 5$ oil through a closed loop system via a centrifugal pump whose heat generation does not adversely affect the refraction. Quartz is used as the solid material inside the test section to enable the index matching. Design of the system is discussed, followed by preliminary results for three rectangular slot flows that coalesce into a single channel. Particle Image Velocimetry was employed to capture the velocities in the mixing region. This configuration allows for simultaneous measurement of the flow 0.35 hydraulic diameters upstream and 3.9 diameters downstream of the outlets. The Reynolds numbers of the three channels are in the range of 1800-20000, spanning multiple flow regimes and promoting different flow physics. Results in the turbulent regime closely resembled the interaction of three confined slot jets. The non-turbulent regime shows the fluid stalling to approximately half of its inlet velocity, prompting recirculation zones near the outlet. The dominant energy contributions and momentum dissipation in the stalling region come from shear and spanwise stresses, whereas streamwise stresses dominate in other locations. It was also observed that the shear stresses decrease with increasing bulk velocities, while the spanwise stresses remained relatively constant. The ability to simultaneously measure upstream and downstream of the outlets enables further investigation into understanding the complex interaction of the momentum exchange that occurs as a result of the geometry, flow regime, and the effect of pressure in these areas. This data also enables a straightforward exercise to help validate models of turbulence and transition for such mixing scenarios.
\end{abstract}

KEY WORDS: Matched index of refraction; Hydraulic mixing; PIV; Turbulent; Laminar; Multi-regime flow.

\section{INTRODUCTION}

In fluid mechanics, mixing occurs when separate fluids with independent properties combine, giving rise to the possibility of momentum, energy, and chemical species exchange with one another. These phenomena are of interest in almost any application involving dynamic fluid domains. The behavior can be simply and qualitatively expressed by introducing two fluid streams, flowing through separate channels. Suppose that each channel has its respective hydraulic, thermal, and chemical properties and that each channel differs in at least one property from the other. Upon their intersection, the blending of the fluids causes the formation of a mixture with combinatory characteristics between the two, creating a new stream whose properties differ from that of the original two, but are a function of both.

Hydraulic mixing is the primary means of momentum exchange and kinetic energy transfer. It is best analyzed qualitatively by understanding the velocity fluctuations within the fluid itself, typically in the form of normal and shear stresses or attempts to measure the kinetic energy itself. Thermal and chemical mixing accommodate the momentum exchange via scalar transport, further complicating the mixing process by

*Corresponding Author: kuaw2006@tamu.edu 
forming a coupled series of interactions and equations. Theoretical knowledge of the coupled behaviour has seen extensive treatment in the literature, but there is still much to gain from empirical means.

One such application involving hydraulic and thermal mixing is the design of the prismatic hightemperature gas reactor (HTGR). Helium coolant flows from many jets of different sizes into the core, mixing and inducing several stresses from the velocity and temperature gradients, which lead to thermal striping in the core $[1,2]$. The hexagonal-shaped core is filled with support posts, further complicating the estimation of the thermal-hydraulic behavior due to the mixing phenomena and complex geometries in the core.

Computational fluid dynamics (CFD) models for the HTGR problem are hence demanding. Not only are high quality, non-isothermal turbulent problems with complex geometries computationally costly, but the experimental data on such flows is limited, translating to high numerical and experimental uncertainties [3]. To reduce these uncertainties, high-quality experimental studies must be carried out to verify and validate the CFD models [4]. A matched index of refraction (MIR) facility was constructed at Texas A\&M University to enable such high-quality experiments. The facility is thus designed with the capability of simulating multiregime flows in separated mixing channels, as discussed in the next section.

\section{MIR FACILITY}

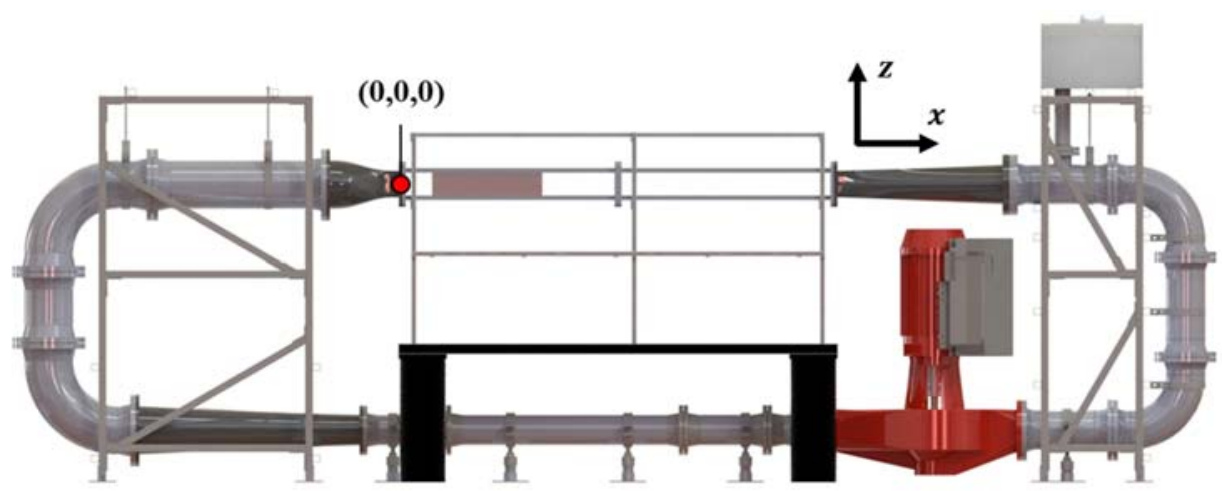

Fig. 1 Front view of the MIR facility (flow direction is clockwise)

The MIR facility shown in Fig. 1 consists of a closed loop tunnel with a $\sim 2.4 \mathrm{~m}$ long optically accessible test section, enabling non-intrusive measurements. The MIR phenomenon is achieved via Drakeol 5 light mineral oil as the working fluid, whose index of refraction (IOR) can be sufficiently matched with that of quartz across all operating temperatures. Although the indices of refraction are sensitive to temperature variations, literature suggests that the IOR mismatch due to a temperature rise of $40^{\circ} \mathrm{C}$ would be necessary to cause a velocity measurement uncertainty of $\pm 0.1 \%$ using a mono particle image velocimetry (PIV) setup [5]. For reference, this would correspond to a IOR mismatch of $0.6 \%$. The temperature dependence is similar for both the quartz and oil indices of refraction, therefore the IOR mismatch remains negligible in most instances. The IOR mismatch is illustrated in Fig. 2 where the mismatch, represented as the percent difference between the indices of refraction, is expected to be below $0.4 \%$ since no test exceeds $30^{\circ} \mathrm{C}$.

The test section consists of a rectangular main channel $(2387.6 \times 50.8 \times 127 \mathrm{~mm})$ with two solid quartz plates separating the flow into two $609.6 \times 14.3 \times 127 \mathrm{~mm}$ outer sub-channels and a $609.6 \times 9.525 \times 127 \mathrm{~mm}$ inner sub-channel. The separating channels are $171.45 \mathrm{~mm}$ downstream of the inlet to the test section, which allows flow measurements prior to separation, while separated, and after mixing. Unlike typical studies where the flow is measured starting at the mixing region [6], the existing setup enables flow measurements to begin well-before the mixing region, providing a cumulative understanding of the mixing phenomenon. This is valuable for both theoretical evaluation and CFD models. The dimensions of the main channel are used to calculate a hydraulic diameter $\left(\mathrm{D}_{\text {hyd }}=72.57 \mathrm{~mm}\right)$. 
TFEC-2020-32419

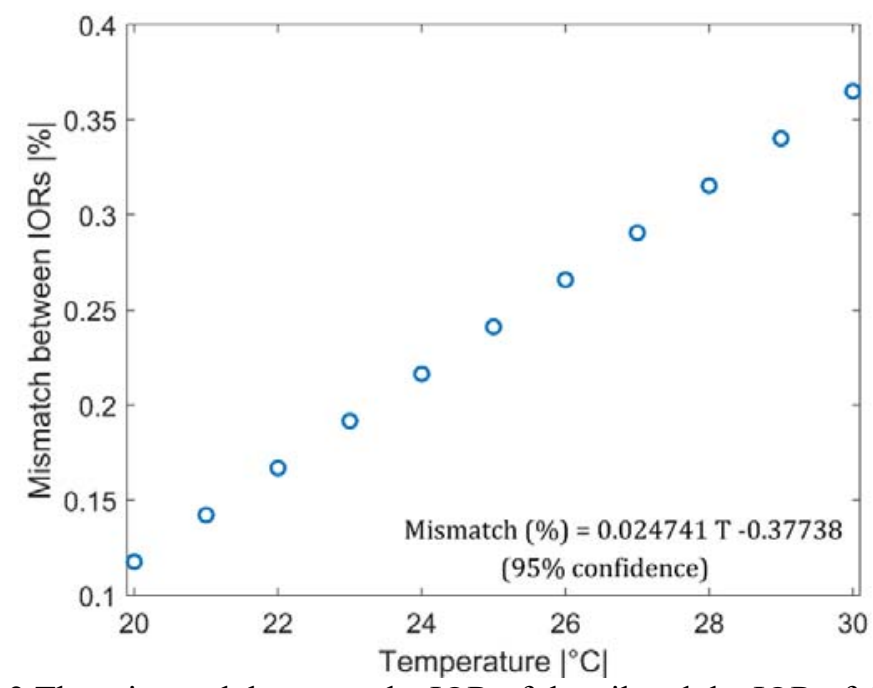

Fig. 2 The mismatch between the IOR of the oil and the IOR of the quartz.

The flow is motivated using a centrifugal pump equipped with a variable frequency drive (VFD) motor, which is capable of simulating inlet bulk velocities between 1.07 and $8.17 \mathrm{~m} / \mathrm{s}$ in the test section, yielding Reynolds numbers between 1800 and 13,000 in the inner sub-channel and 2700 and 20,000 in the outer sub-channels. Although the facility is not currently equipped with a temperature control system, characterizing its temperature behavior in its current state enables experiments to account for any temperature variations that may occur. Hourlong temperature tests were conducted for multiple operating VFD frequencies using a type $T$ thermocouple, where the temperature rise was linear throughout the hour. The slope at which the temperature increases at each frequency represents the temperature rise as a function of time, which is shown in Fig. 3.

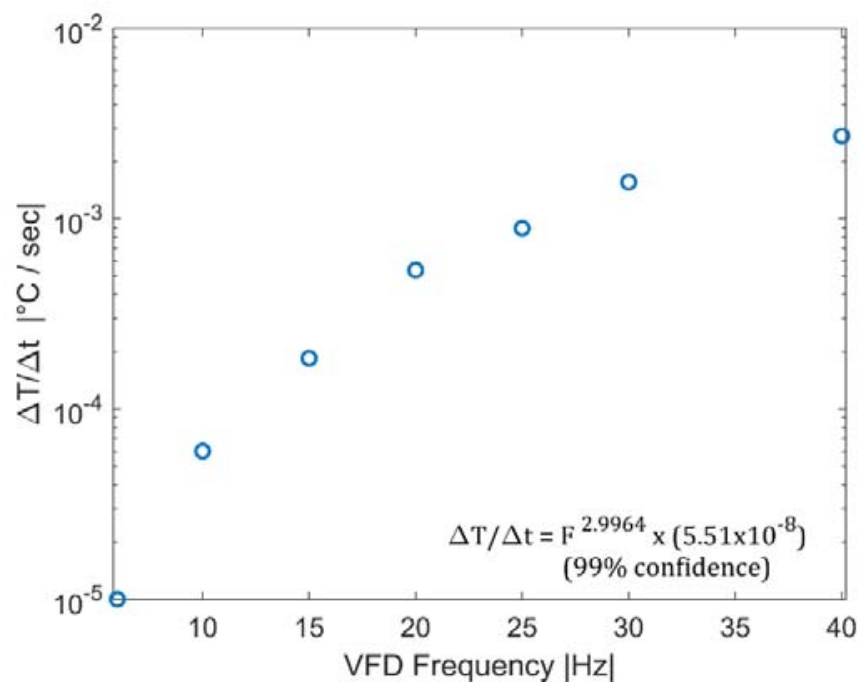

Fig. 3 The rate of temperature rise over time at different VFD settings, enabling an understanding of the temperature behavior in the facility without a temperature control system.

The velocity behavior was characterized through a set of PIV tests at the inlet of the test section with 10 different VFD settings. Tests were conducted in the height-wise or transverse plane $(x z)$ and the spanwise plane $(x y)$. Repeatable tests were conducted with the lowest and highest VFD settings, each spanning 6 trials. The centerline inlet profiles for the repeatable tests in the $x z$-plane and the $x y$-plane are shown in Fig. 4(a) and Fig. 4(b) respectively, provided at VFD frequencies to illustrate the operation limits of the facility. The inlet bulk velocities for the $x z$ and $x y$-planes respectively are shown in Fig. 5(a) and Fig. 5(b) as a function of the VFD 
input levels. It should be noted that the measurement location in these studies is taken at $\sim 1.25$ hydraulic diameters from the physical inlet of the test section, or $\sim 1.1$ hydraulic diameters upstream of the leading edge of the quartz plates. Therefore the velocities in Fig. 4 and Fig. 5 represent those before the initial separation into the three channels.

Given the operational control and limits of the facility, it is worth noting that the Reynolds number can be adjusted to encompass multiple flow regimes. For the inner channel, a Reynolds number as low as 1800 is achievable. With the upper limit of Reynolds number at 20,000, a wide variety of hydraulic mixing experiments are feasible, spanning laminar, transition, and turbulent flow regimes. Further details on the facility including the pressure drop in the system can be found in [7].

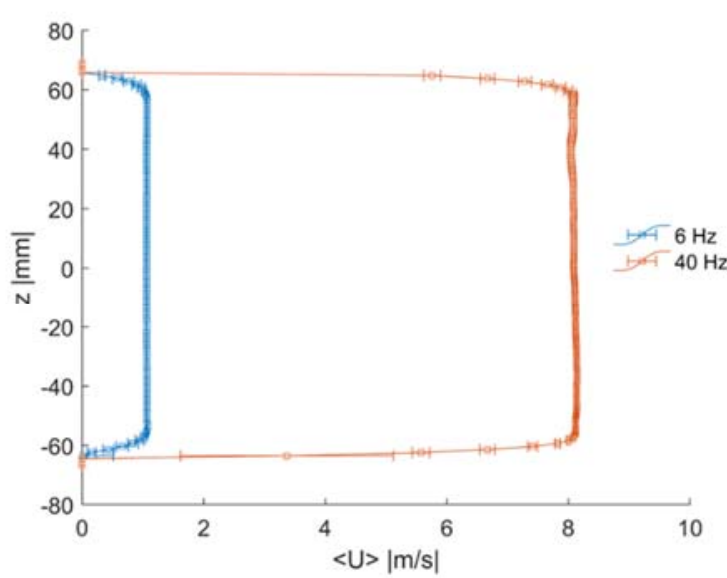

(a)

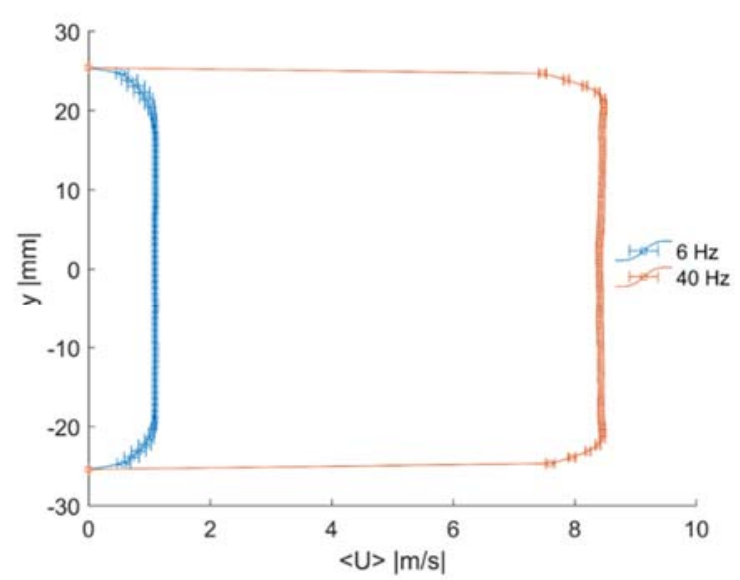

(b)

Fig. 4 The centerline inlet profiles for the minimum and maximum VFD settings in the (a) transverse plane and (b) the spanwise plane.

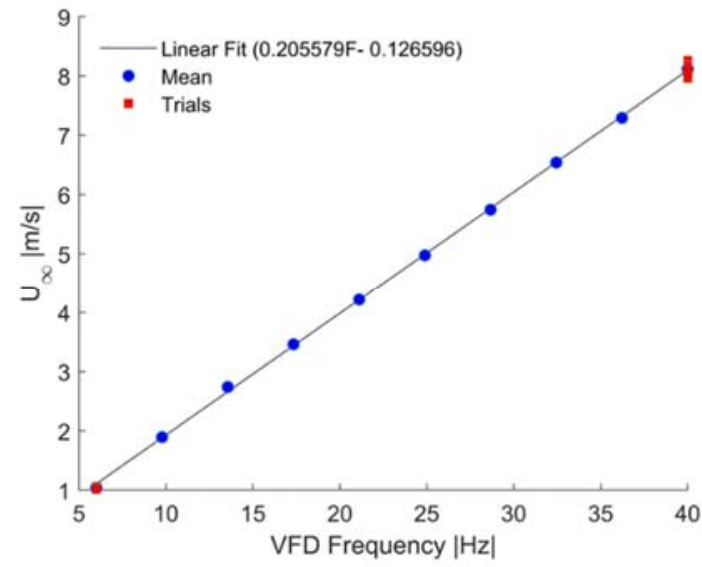

(a)

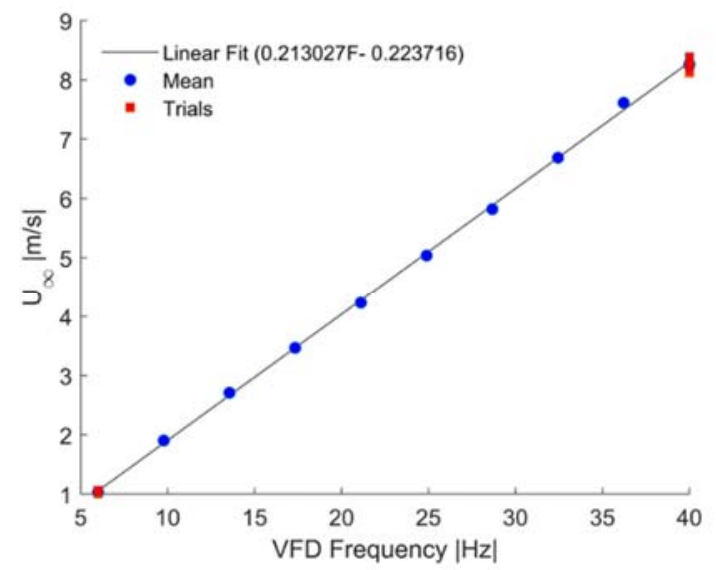

(b)

Fig. 5 The inlet space averaged bulk velocities for all VFD settings in the (a) transverse plane and (b) the spanwise plane.

\section{HYDRAULIC MIXING EXPERIMENT}

Hydraulic mixing tests are conducted with multiple inlet bulk velocities $\left(U_{\infty}\right)$ at $1.07,1.86,2.65$, and 3.44 $\mathrm{m} / \mathrm{s}$. This enables the measurements at various Reynolds numbers, each corresponding to a different flow regime. The inlet bulk velocity and the hydraulic diameter are used to non-dimensionalize the results. Velocity profiles are 
obtained using a mono PIV setup that captures profiles $0.35 \mathrm{D}_{\text {hyd }}$ upstream and $3.9 \mathrm{D}_{\text {hyd }}$ downstream from the outlet of the channels. To obtain large spatial profiles, four Phantom Micro M120 cameras are utilized with Nikon $52 \mathrm{~m}$ $\mathrm{f} / 1.8 \mathrm{D}$ lenses, and LaVision Inc. $527 \mathrm{~nm}$ lens filters. A dual-head DM30-527 Argon laser with a wavelength of $527 \mathrm{~nm}$ is utilized to generate a $\sim 1 \mathrm{~mm}$ thick laser sheet cutting through the $x y$-plane. Each camera is also equipped with Scheimpflug mounts to fine-tune the field of view and ensure it is normal to the laser sheet. The measurement setup is optimized to obtain a temporal resolution of $600 \mathrm{~Hz}$ and a spatial resolution of 11.524 pixels $/ \mathrm{mm}$. LaVision DaVis 8.4 software is used to process the raw images and obtain the velocity profiles with a 6-pass crosscorrelation with 2 initial passes with a grid size of $64 \times 64$ and 4 more passes with a grid size of $32 \times 32$ with $75 \%$ overlap. Flow temperatures are also measured during testing to verify temperature conditions were stable, and yielded temperatures between $23.45^{\circ} \mathrm{C}$ and $24^{\circ} \mathrm{C}$ across all tests. Fig. 6 shows the spatial velocity profile of the lowest velocity case at $U_{\infty}=1.07 \mathrm{~m} / \mathrm{s}$, where the inner sub-channel and the two outer sub-channels exhibit Reynolds numbers of $\sim 1835$ and $\sim 2660$ respectively. By way of comparison, the Reynolds number after mixing is 5637. As one would expect, the velocity field slows down from a global perspective since the flow area increases downstream of the quartz plates, but the deceleration of the flow in the center sub-channel is more extreme than the two outside sub-channels. The inner sub-channel decelerates to nearly half of the inlet velocity around $\mathrm{x} / \mathrm{D}_{\text {hyd }}$ $=10.8$. This behavior is not unexpected when considering the fact that two mixing layers exist just downstream of the quartz plates. The center channel is sandwiched between these mixing layers, which extract energy from the center mean flow, thereby decreasing the kinetic energy. Of course, energy is also extracted from the top and bottom means flows as well, but each only border a single mixing layer. In addition, their kinetic energy is much higher to start with, and therefore do not see as extreme of a deceleration when compared to the center channel. Another feature worth highlighting is the near zero velocity region immediately downstream of each quartz plate ( $\mathrm{x} / \mathrm{D} \sim 10.4$ and $\mathrm{y} / \mathrm{D} \sim+/-0.1)$. The abrupt end of the plates establishes a condition where separation occurs. The result is a recirculation condition in those regions.

Whereas the streamwise normal stresses $\langle u u\rangle$ are expectedly the dominant energy contributors everywhere in the profile, an exception can be made in the mixing region immediately after the flow recombines. From Fig. 6, this mixing region appears to extend from $\sim 10.4 \mathrm{D}_{\text {hyd }}$ to $\sim 11.5 \mathrm{D}_{\text {hyd }}$ where the flow begins accelerating to match the bulk velocity. Within this region, the shear stresses $\langle u v\rangle$ are present and the spanwise normal stresses $\langle v v\rangle$ dominate the energy contributions. This is most clearly represented at $x=10.67 \mathrm{D}_{\text {hyd }}$ as illustrated in Fig. 7 and Fig. 8 for $\langle u v\rangle$ and $\langle v v\rangle$ respectively. The $\langle u v\rangle$ stresses shown in Fig. 7 are significant only in the lowest Reynolds number case, and gradually decrease with increasing bulk velocities. This is likely due to the recirculation zones illustrated in Fig. 6, which are especially prominent with lower bulk velocities. The $\langle v v\rangle$ stresses on the other hand remain a dominant energy contributor in the mixing region throughout all cases. As illustrated in Fig. 8, the energy contributions from the $\langle v v\rangle$ stresses remain on the same order of magnitude across all cases as opposed to the ever-decreasing $\langle u v\rangle$ stresses with increasing bulk velocities. This is expected as the mixing prompts the flow from one sub-channel to move in non-streamwise directions to combine with the other flows. In this instance, the flow moves in the spanwise direction leading to increased $\langle v v\rangle$ stresses. This suggests that there could also be noteworthy energy contributions in the transverse $(z)$ direction as well. 


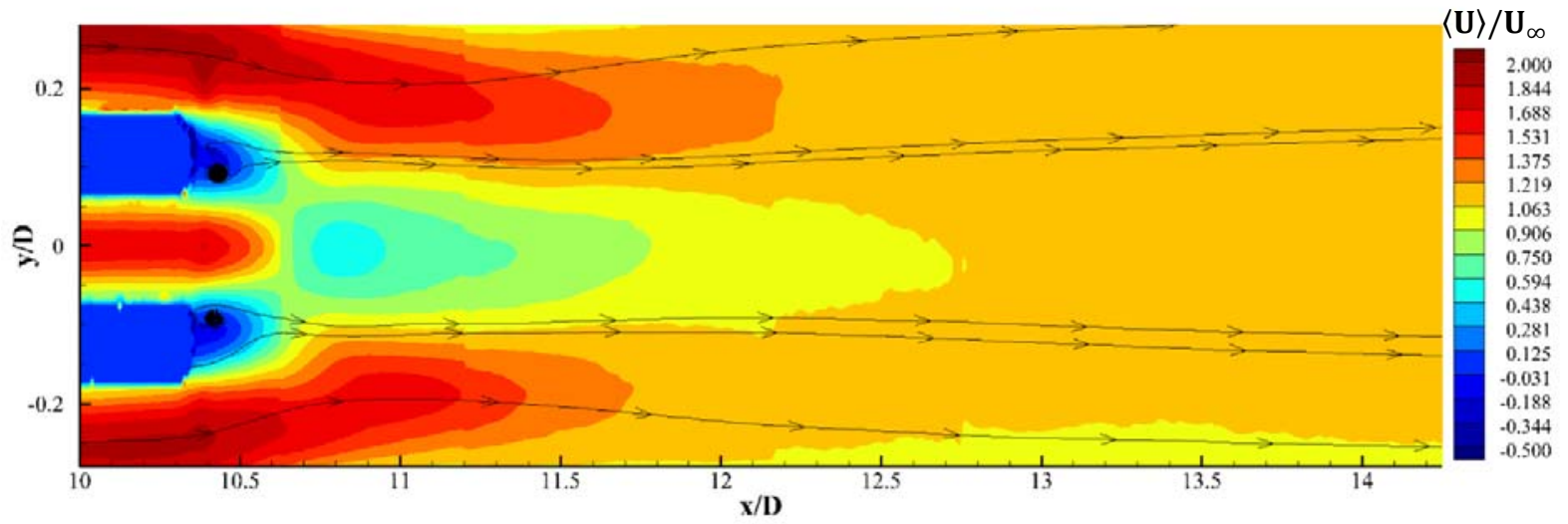

Fig. 6 The spatial velocity profile of ensemble averaged streamwise velocities, where $D=D_{\text {hyd }}$.

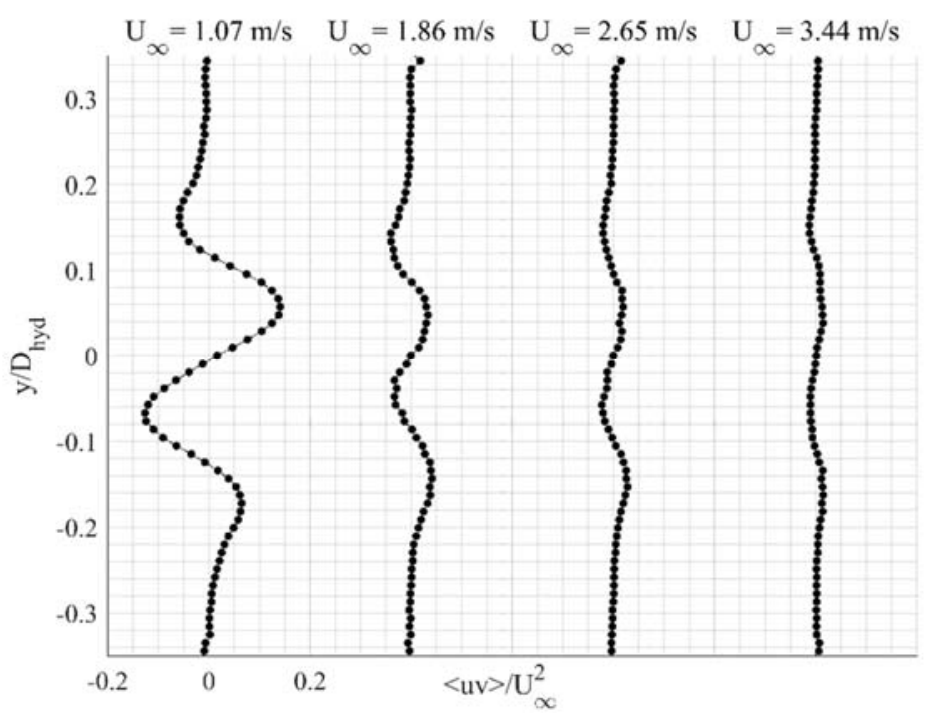

Fig. 7 The shear stress $\langle u v\rangle$ at $10.67 D_{\text {hyd }}$ for each test case.

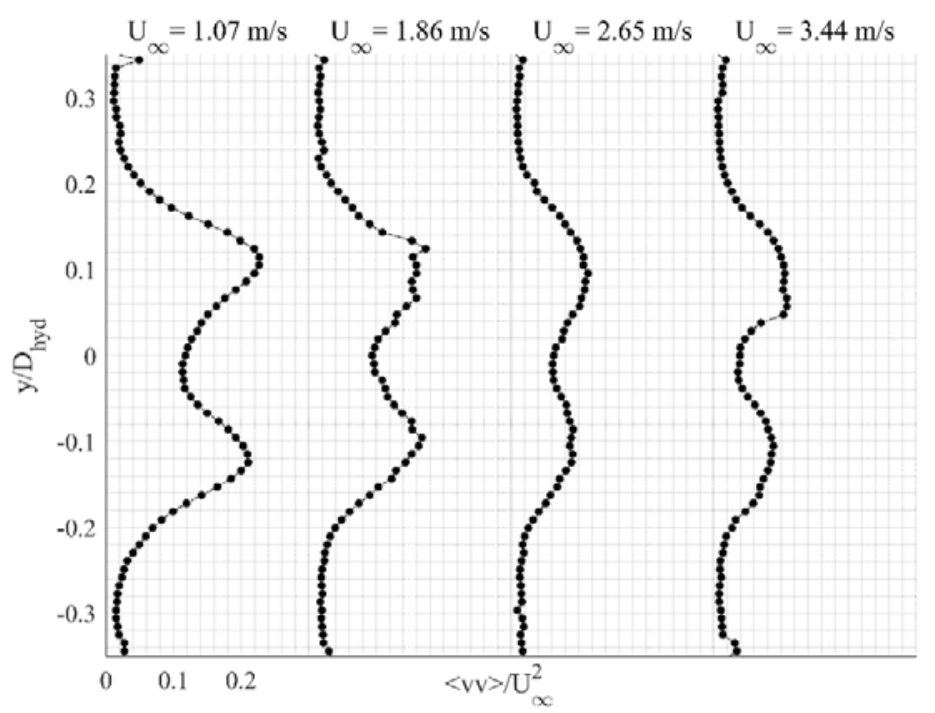

Fig. 8 The spanwise normal stresses $\langle v v\rangle$ at $10.67 \mathrm{D}_{\text {hyd }}$ for each test case. 


\section{CONCLUSIONS}

The recently constructed MIR facility was successfully characterized with multiple temperature and velocity tests, providing a complete assessment of the behavior and capability of the facility. The IOR mismatch was anticipated to be very small based on previous studies [5], suggesting negligible effects on the velocity measurements. The inlet velocities showed flat profiles at the inlet in both the transverse and spanwise planes. The temperature behavior showed a linear increase in temperature with respect to time at every VFD setting, enabling the rate of change in temperature at each VFD setting to be quantified. The full understanding of these trends enabled multiple tests with different flow conditions and regimes without the use of a temperature control system.

Preliminary mixing studies were conducted for three rectangular channels combining into a single channel using a mono PIV setup, where the flow was captured upstream and downstream from the exits of the channels. Results showed significant stalling in the mixing region at the lower Reynolds number regime, while the higher regimes showed more uniform mixing as depicted by lower magnitudes of shear stresses and spanwise normal stresses. Additionally, recirculation zones were observed at the lowest Reynolds number regime, which could hint at the presence of a prominent vortex shedding frequency. Further investigation of the mixing region could result in correlations between the Strouhal numbers and the mixing behavior. Finally, the implication of lower shear and spanwise normal stresses at higher Reynolds numbers leads to the observation that the turbulent kinetic energy, which includes the $\langle u v\rangle$ and $\langle v v\rangle$ stresses, decreases with increasing $U_{\infty}$. This could mean that operating at higher jet stream velocities in an HTGR might help reduce the stresses induced in the core, however further studies are needed. The authors recommend considering temperature control to help introduce thermal effects on the future mixing studies, and different channel sizes and geometries to investigate the geometrical effects on energy dissipation in mixing profiles.

\section{NOMENCLATURE}

$\begin{array}{llllll}U_{\infty} & \text { Inlet bulk velocity } & (\mathrm{m} / \mathrm{s}) & \langle u u\rangle & \text { Streamwise normal stress } & \left(\mathrm{m}^{2} / \mathrm{s}^{2}\right) \\ x & \text { Streamwise direction } & (\mathrm{mm}) & \langle u v\rangle & \text { Shear stress } & \left(\mathrm{m}^{2} / \mathrm{s}^{2}\right) \\ y & \text { Spanwise direction } & (\mathrm{mm}) & \langle v v\rangle & \text { Spanwise normal stress } & \left(\mathrm{m}^{2} / \mathrm{s}^{2}\right) \\ z & \text { Transverse direction } & (\mathrm{mm}) & & & \end{array}$

\section{REFERENCES}

[1] Salkhordeh, S., Clifford, C., Jana, A., Kimber, M. L., "Large Eddy Simulations of scaled HTGR lower plenum for assessment of turbulent mixing," Nuclear Engineering and Design, 334, pp. 24-41, (2018).

[2] Tokuhiro, A., Kimura, N., "An experimental investigation on thermal striping: Mixing phenomena of a vertical non-buoyant jet with two adjacent buoyant jets as measured by ultrasound Doppler velocimetry," Nuclear Engineering and Design, 188, pp. 49-73, (1999).

[3] Colmenares, J.D., Poroseva, S., Peet, Y. T., Murman, S. M. "Analysis of uncertainty sources in DNS of a turbulent mixing layer using NEK5000,” CFD Modeling and Applications II 2018, 2018 Fluid Dynamics Conference., (2018).

[4] Landfried, D. T., Kristo, P., Clifford, C. E., Kimber, M. L., "Design of an experimental facility with a unit cell test section for studies of the lower plenum in prismatic high temperature gas reactors," Annals of Nuclear Energy, 133, pp. 236-247, (2019).

[5] Sabharwall, P., Conder, T., Skifton, R., Stoots, C., Kim, E. S., PIV Uncertainty Methodologies for CFD Code Validation at the MIR Facility. Retrieved July 1, 2019, from https://inldigitallibrary.inl.gov/sites/sti/sti/5901284.pdf, (December 2013).

[6] Alwafi, A., Nguyen, T., Hassan, Y., Anand, N. K., "Time-resolved particle image velocimetry measurements of a single impinging jet in the upper plenum of a scaled facility of high temperature gas-cooled reactors," International Journal of Heat and Fluid Flow, 76, pp. 113-129, (2019).

[7] Reyes-Gonzalez, J. R., Design and Construction of a High-speed Matched Index of Refraction Facility, Master's Thesis, Texas A\&M University, (2018). 